\title{
A Potential Biomarker MicroRNAs in the Diagnosis of Some Psychiatric and Neurodegenerative Disorders
}

\section{Bazı Psikiyatrik ve Nörodejeneratif Bozuklukların Tanısında Potansiyel Bir Biyobelirteç MikroRNA' ların İncelenmesi}

\author{
Fatma Akat', @Hüsamettin Vatansev², @Derya Karaoğlu Gündoğdu³ \\ 'Selcuk University Institute of Health Sciences Department of Medical Biochemistry, Konya, Turkey \\ ${ }^{2}$ Selcuk University School of Medicine, Department of Medical Biochemistry, Konya, Turkey \\ ${ }^{3}$ Selcuk University Faculty of Medicine, Department of Surgical Medical Sciences, Department of Brain and Nerve Surgery, Konya, Turkey
}

\begin{abstract}
MicroRNAs (miRNAs) are a large endogenous, noncoding RNA class of 19-25 nucleotides. Mature miRNAs regulate target gene expression at both transcriptional and translational levels. As a current area of interest in molecular diagnostic research, microRNAs (miRNAs) have recently been shown to be effective circulatory biomarkers for many diseases. Major depressive disorder, Bipolar disorder, Schizophrenia, Obsessive compulsive disorder, Alzheimer's disease and Parkinson's disease are among the most common psychiatric and neurodegenerative disorders known to be a serious public health problem in terms of morbidity, mortality and functional handicap. MicroRNAs are aware of the events occurring in the cell as normal mechanical, physical and biochemical functions due to many psychiatric and other medical diseases, and are very promising as a biomarker for emerging disorders and neuropathological changes.
\end{abstract}

Keywords: Biomarker, miRNA, psychiatry, neurodegenerative disorder

\section{Öz}

Mikro Ribonükleik asitler (miRNA'lar) 19-25 nükleotitin büyük bir endojen, kodlayıcı olmayan RNA sınıfıdır. Olgun miRNA'lar hedef gen ekspresyonunu hem transkripsiyonel hem de translasyonel seviyelerde düzenler. Moleküler teşhis araştırmalarına yönelik güncel bir ilgi alanı olarak, mikroRNA'ların (miRNA'ların) son zamanlarda birçok hastalık için etkili dolaşım biyobelirteçleri olduğu gösterilmiştir. Majör depresif bozukluk, Bipolar bozukluk, Şizofreni, Obsesif kompulsif bozukluk, Alzheimer hastalığı ve Parkinson hastalığı günümüzde morbidite, mortalite ve fonksiyonel handikap açısından ciddi bir halk sağlığı sorunu oluşturduğu bilinen en yaygın psikiyatrik ve nörodejeneratif bozukluklar arasındadır. MikroRNA'lar psikiyatrik ve diğer tıbbi birçok hastalık nedeniyle normal mekanik, fiziksel ve biyokimyasal işlevler olarak hücre içinde gerçekleşen olaylardan haberdar olup, ortaya çıkan bozukluklar ve nöropatolojik değişiklikler için bir biyobelirteç olma bakımından oldukça umut vadetmektedir.

Anahtar Kelimeler: Biyobelirteç, miRNA, psikiyatri, nörodejeneratif bozukluk 


\section{INTRODUCTION}

Biomarkers have been used in clinical medicine for decades. Biomarker is a feature that can be measured objectively as an indicator of normal or pathological processes and pharmacological response to a therapeutic intervention. ${ }^{[1]}$ It is classified according to its applications. These; diagnostic biomarkers for early diagnosis of the disease, screening biomarkers referring to seeking illness in people with no signs of disease, prognostic biomarkers to predict the course of the future disease and evaluate the clinical response to an intervention, and finally staging biomarkers to determine the severity and progression of the disease..$^{[2]}$

As a current area of interest in molecular diagnostic research, miRNAs have recently been shown in the literature to be effective circulatory biomarkers for many diseases. These small non-coding RNAs, which regulate the sequence of many genes, are also involved in various biological processes in the central nervous system (CNS) ${ }^{[3]}$

MiRNAs are a large endogenous, non-coding RNA class of 19-25 nucleotides. ${ }^{[4]}$ Since $1990 \mathrm{~s}$, miRNAs, small noncoding RNAs, have been acting as key-key transcriptional regulators of RNAs that encode protein in multicellular eukaryotes, including fungi, plants, and animals. A key feature of miRNAs is the high level of evolutionary protection among species compared to other non-coding RNAs, and these properties show their importance in the physiology of multicellular organisms. This protection has been effective in identifying genes regulated by miRNA and in investigating their contribution to human disease pathophysiology in animal models for human diseases..$^{[5]}$ miRNA has significant regulatory capacity and has been shown to be vital for the flow of a number of neural processes through the regulation of neurogenesis and migration timing through the regulation of neurotransmission and synaptic plasticity. ${ }^{[6]}$ miRNAs are embedded in complex regulatory networks because a single miRNA can regulate hundreds of target mRNAs that encode different proteins. More than a thousand miRNAs are predicted to be associated with pathological conditions, suggesting that miRNAs play an important role in many biological processes during health and disease. ${ }^{[4]}$ miRNAs play a role in many physiological functions. Some of these are cell growth, differentiation, stress response and death, apoptosis and autophagy, cancer metastasis, angiogenesis, tissue repair. In addition to the most frequently studied cancer cases for miRNAs; insulin resistance and diabetes, coronary artery disease, stroke, ischemic kidney injury and many more complex disorders have also been shown to play a role in the pathophysiology. ${ }^{[5]}$ Over the past decade, irregularity and variability in proteins processed by various miRNAs have been found to be associated with neurodegenerative disorders and neuroblastoma. miRNA rate of autism spectrum disorders, schizophrenia, has been found to vary in the developmental brain disorders such as mental impairment and epilepsy. They also play a role in various neurodegenerative diseases such as
Alzheimer's disease, Huntington's disease, Parkinson's disease, amyotrophic lateral sclerosis. However, the exact mechanism of miRNA in brain development, learning, memory, and highlevel brain processes is still not fully understood. ${ }^{[7]}$ miRNAs; fine regulation of genes associated with pathologies can have an important mechanism to maintain neuronal balance and ensure that neuronal circuits respond adequately to environmental insults. ${ }^{[8]}$ As an example of the great effect on gene expression, miRNAs are heavily involved in the early stages of development when pluripotent cells switch to soy-specific cells. This change is caused by upregulation of miRNAs, among other factors. In particular, miR-9 and miR-124a are vital for neural development; they are highly expressed by neurons and astrocytes in the brain and regulate the phosphorylation of STAT3, an important molecule for neuronal differentiation. ${ }^{[4]}$ Through reversible changes in gene expression, miRNAs can cause CNS to malfunction when faced with extreme environmental stimuli. Evidence supports the pathological role of miRNAs in reshaping neural information processing in many neuropsychiatric events, including major depressive disorder, bipolar disorder, and schizophrenia. Recent evidence suggests the role of miRNAs as a dynamic interface to interact with the internal information processing system reflected by expression changes in the brain. ${ }^{[9]}$ miRNAs are known to be packaged, especially in small organelles such as exosomes and extracellular fluids. Packed in this way, extracellular miRNAs are protected from exonucleases and can survive for a long time in body fluids ${ }^{[5]}$ miRNAs can travel in biological fluids (serum, plasma, urine, cerebrospinal fluid and saliva) and promote their endocrine gene regulation by performing their activities in distal cells or tissues. Therefore, circulating miRNAs must ensure their stability in order to pass through biological fluids. This is largely accomplished by the association of miRNAs in exosomes and high-density lipoproteins, preserving and preventing disruption before reaching the target cells. ${ }^{[10]}$ miRNA expression changes in the peripheral circulation (in cells such as plasma, serum, and non-neuronal tissue or lymphocytes) have been found to be highly associated with changes in neuronal tissue in patients with various neuropsychiatric disorders. ${ }^{[9]}$ Exosomal miRNAs are also found in human cerebrospinal fluid (CSF) and are recommended as biomarker and/or drug targets for neuropsychiatric disorders such as temporal lobe epilepsy. In particular, exosomal packaged miRNAs are associated with neuronal depolarization as well as cellular communication between neurons and glia. Therefore, in addition to being key regulators of gene transcription, miRNAs also function in tissue communication with an action mechanism such as peptide and lipid

Neurodegenerative diseases are a group of diseases caused by chronic and progressive degeneration of neural tissue. The main pathological findings are neuronal degeneration and neuron loss in the brain and/or spinal cord. ${ }^{[1]]}$ Psychiatric and neurodegenerative disorders are conditions that have negative effects for both the individual and the society; in 
addition to bringing heavy health and economic burden to the society, it significantly impairs the normal functioning and quality of life of the person. Despite these facts, there is limited pharmacotherapy to treat these disorders, and most treatments are inadequate in terms of effectiveness and tolerability. One of the obstacles in the treatment of these multi-factor, complex and heterogeneous disorders; the genetic architecture of these disorders is not yet fully elucidated. ${ }^{[12]}$ Molecular intervention or disruption of neuronal miRNAs in model systems is an important strategy to understand the effect of irregularities in gene expression networks in psychiatric disorders. ${ }^{[6]}$ The purpose of this review is; to talk about the role and importance of miRNAs in synaptic plasticity and CNS neurogenesis and to review the studies with some psychiatric and neurodegenerative disorders systematically with current literature reviews.

\section{miRNA Biogenesis}

RNAs are generally classified according to their nucleotide lengths. ${ }^{[13]}$ miRNA is a non-coding RNA that has a role in modulating the coding potential of a replicated messenger RNA (mRNA) based on characteristic sequence complementarity.

In the first report as an epigenetic modifier, miRNA is thought to work as a modulator of a single protein coding gene, while today it is known to act as a potential regulatory center in controlling a complex gene network either directly through a protein coding gene or indirectly. ${ }^{[9]}$ Epigenetic events are important gene action modifiers and causes of various human diseases. One of the main mechanisms of such actions is various expression of miRNAs. One of the important features of miRNA among the many functions described is that they are gene expression regulators. Non-coding RNAs, especially miRNAs, have been shown to play an important role in epigenetic control of gene expression in various organs, including the uterus. miRNAs are copied from genes scattered across all chromosomes, except the Y chromosome. ${ }^{[13]}$ Although small molecules and have the potential to go through exon-coupling to create more structural diversity, miRNAs show functional diversity by targeting various RNA molecules ranging from protein-encoding (mRNA) to noncoding RNAs. In humans, up to now $\sim 2500$ mature miRNAs have been disclosed, and about 30\% of them have been found in intronic regions of protein coding genes, sometimes promoting the facultative use of host gene transcription machines. However, usually miRNA loci are mapped alongside different transcriptional units that mostly use RNA polymerase II for transcription. ${ }^{[9]}$

As shown in Figure 1, miRNAs are processed from primary miRNA (pri-miRNA) transcripts, most of which are copied from independent miRNA genes by RNA polymerase II, while others are found in the introns of protein-coding genes. ${ }^{[14]}$ The main route in miRNA biogenesis is the canonical path. In this way, it all starts in the cell nucleus, where the miRNA gene is copied by RNA polymerase II/III to the primary miRNA, which is a hairpin ring. ${ }^{[13]}$ Within cell nuclei, immediately after transcription, pri-miRNAs are separated into precursor miRNAs by Drosha ribonuclease III (DROSHA) and microprocessor complex subunit DGCR8. ${ }^{[15]}$ All other processes are located outside the nucleus because this leading miRNA molecule is transported into the cytoplasm using exportin 5 . The leading miRNA is then processed into a duplex by the endoribonuclease Dicer and RNA binding protein (TRBP). Finally, this duplex is separated and forms the mature, singlestranded and functional miRNA molecule. $5 p$ or $3 p$ strands of mature miRNA duplex are incorporated into Argonaute proteins (AGO) to form an RNA-induced squelch complex (RISC). This complex will be responsible for translational inhibition by translational suppression or mRNA degradation. [13] The seed region consists of two to seven nucleotides of the miRNA sequence and is the most critical region for target recognition. When excellent complementarity exists between the miRNA and the target mRNA, the target mRNA decreases. In the absence of perfect complementarity, the target is "deadenylated", resulting in the depletion of the target mRNA and subsequent exonucleolytic digestion or translational suppression (a different mechanism at each translation step, ie initiation, elongation and termination). [12] Mature miRNAs regulate target gene expression at both transcriptional and translational levels. ${ }^{[13]}$ Given the key role of miRNAs in mediating biological events, any disruption in their expression may cause impaired homeostasis, which is reflected as an imbalance in the regulatory network that can distinguish between normal and disease states. ${ }^{[9]}$

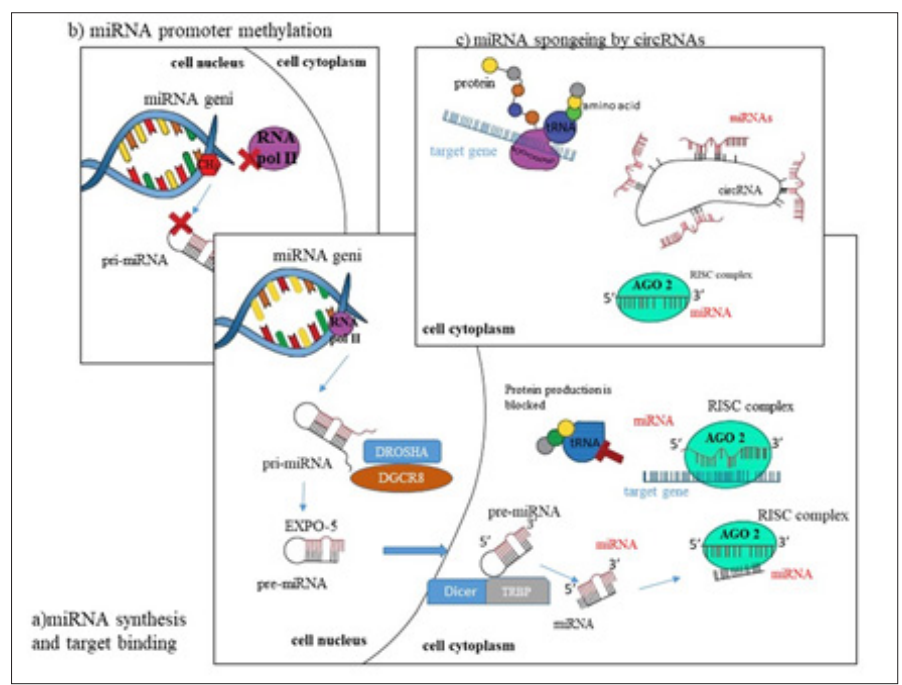

Figure 1. miRNA Biogenesis

\section{The Plasticity Role of miRNAs}

Neural networks display adaptations of their activities that limit the strength of synaptic connections. Such neural network adaptations with synaptic plasticity are thought to be a cellular learning and memory substrate. Synaptic plasticity has been extensively studied in the hippocampus, as this region plays a critical role in long-term memory formation. ${ }^{[16]}$ Increasing evidence suggests that miRNAs 
are involved in neurogenesis, synapse development, axon guidance, and regulation of neuronal plasticity in both developing and adult brains..$^{[9]}$ Among the first clues for this role are the observations that miRNAs in neurons mate with polyribosomes and are often found in dendrites. ${ }^{[5]}$ Irregularity in synaptic plasticity represents the inability of the central nervous system to properly integrate various neuronal inputs that make adaptive changes in neurons to promote appropriate response to external stimuli.. ${ }^{\left[{ }^{[9]}\right.}$ Disruption of $\mathrm{N}$-methyl-D aspartate (NMDA) glutamate receptor signal reduced levels of behavioral responses of modulated impaired NMDA receptor delivery of miRNA, miR-219 and miR-219 in the mouse prefrontal cortex. Argonaute protein Ago2 combines with miRNAs to target specific mRNAs; Rapid changes in Ago2 phosphorylation were observed in NMDA-mediated dendritic spine morphogenesis following NMDA receptor stimulation affecting miRNAs. ${ }^{[5]}$ Many other miRNAs have been identified as they play a role in regulating plasticity-related functions in the brain. These include miR-9, miR-125a/b, and miR-188. Individually, these miRNAs are associated with a gene cell (eg REST, FXR1P, CAMKK2-AMPK, PSD-95, BCL-W, SYN-2, NRP-2, 2-AG and BACE) directly or indirectly associated with synaptic plasticity may affect epigenetically. ${ }^{[9]}$ Deficient neuroplasticity is considered to be a major cause, especially in the pathology of neuropsychiatric disorders, especially mood disorders, schizophrenia and autism spectrum disorder. Therefore, it is evident that in these disorders, the abnormal regulation of brain miRNA transcription as well as the secretion of exosomal miRNAs involved in neuron-glia communication have changed. ${ }^{[5]}$

\section{miRNAs and Major Depressive Disorder}

Major depressive disorder (MDD) is a chronic, debilitating and life-threatening most common psychiatric disorder. Psychomotor is characterized by at least 2 weeks of depressive mood associated with agitation or slowing, guilt, suicidal behavior, changes in appetite and/or weight, sleep patterns, changes in attention, concentration and energy. ${ }^{[14]}$ It is estimated that $30-40 \%$ of the risk of depression is caused by genetic factors. More than 264 million people worldwide suffered from major depression in 2019, and about 800,000 people die annually due to suicide. ${ }^{[4]}$ In MDD, more than 25 important reports showed miRNA-related expression changes in many vital brain areas (anterior cingulate cortex, Brodmann's area [BA] 9, BA10, BA44, BA46 and locus coeruleus $[L C])$. The expression of miR-508-3p and miR-152-3p was significantly downregulated in the major depressive disorder brain compared to control subjects; however, miR-508-3p expression was significantly lower in suicide subjects than in non-suicide subjects. ${ }^{[9]}$ It reported lower expression levels of miR-135 in blood samples from depressed patients, and their levels are associated with antidepressant drug activity. In particular, miR-135 along with miR-135 has been shown to be essential for maintaining NMDA receptor dependent dendritic backbone reconstruction. Among miRNAs that are reported to be irregular in MDD, miR-1202 stands out as primate-specific miRNA (without homologous): Lower levels of miR-1202 were found in both blood and post-mortem brain tissues of MDD patients..$^{[5]}$ Recently, in a case report, depression patients have been reported to have lower levels of brain-derived neurotrophic factor (BDNF) and increased expression of microRNA-132 and microRNA-182 compared to healthy subjects. It has been emphasized that serum BDNF and related miRNAs can be used as biomarkers for diagnosis or as therapeutic targets of depression. ${ }^{[10]}$ Independent of antidepressant use in the acute depressive phase of MDD, plasma miR-134 downregulation was present, plasma miR134 levels increased with symptomatic improvement after an 8-week antidepressant treatment period, and subjects with the lowest miR-134 concentration may not respond to conventional antidepressants. ${ }^{[3]}$ Many studies have investigated changes in miRNA systems in patients diagnosed with MDD or during major depressive episodes. Overall, the findings obtained so far have rarely been repeated between studies, possibly due to the heterogeneity of samples. In several studies, only a few miRNAs have been reported to change; among them, miR-132, miR-451a and miR-34a-5p seem to be the most consistent. In addition, let7 $\mathrm{b}$ is important for miR-182, miR-124, miR-345, miR-146b-5p, miR-146a, miR494, miR-376a, miR-107, miR-33a and miR-221-3p replication of the findings was observed. ${ }^{[14]}$ The clinical significance of this miRNA (miR-34a-5p) is suggested to be evaluated for evidence of a positive correlation between miR-34a-5p levels and the severity of depressive symptoms, thus showing significant relationships with the history of the disease and suicide attempt. Similar to miR-451a, miR-34a-5p levels have been shown to show relatively high specificity (95.24\%) and sensitivity (96.88\%) values for MDD diagnosis; which is particularly exciting for the future use of miRNA as a potential biomarker. ${ }^{[14]}$

\section{miRNA and Schizophrenia}

Schizophrenia is a fairly common neuropsychiatric disorder that affects about $1 \%$ of the population worldwide. Research findings have shown that genetic factors are powerful mediatorsforthe development ofschizophrenia, as theheredity of schizophrenia is estimated to vary from $24-80 \%$ depending on the endophenotype. Schizophrenia is characterized by positive (eg hallucinations, delusions), negative (eg anxiety, depression) and cognitive (eg memory impairments) symptoms, which leads to a difficult heterogeneous disease that is difficult to diagnose. ${ }^{[4]}$ To analyze the diagnostic potential of miRNAs as specific biomarkers in peripheral blood mononuclear cells, the nine most frequently reported miRNAs associated with schizophrenia (miR-30e, miR-34a, miR-181b, miR-195, miR-346, miR- 432, miR-7, miR-132 and miR-212) expression levels were analyzed in schizophrenia patients and healthy controls. It has been revealed that there are increased expressions of miR-212, miR-34a and miR-30e in patients with schizophrenia compared to controls. The sensitivity and specificity of miR-30e as the diagnostic biomarker were $81.80 \%$ 
and $68.00 \%$, respectively. These evaluations were also made on the plasma sample. Findings showed higher significance of abnormal miRNA expression in plasma (at $90.90 \%$ and $60.00 \%$ sensitivity, respectively) between patients with schizophrenia and normal controls, suggesting that miR-30e may be a more sensitive biomarker in the diagnosis of schizophrenia. ${ }^{[2]} \mathrm{miR}$ 137 overexpression affects synaptogenesis, pre-synaptic microstructure and function, thereby reducing synapse density, a potential central disruption in schizophrenia, and compromising synapse performance. Regulates genes involved in neurodevelopment, including down-regulated miR-132, DNMT3A, DPYSL3 and GATA2. ${ }^{[17]}$ Indeed, high miR137 plasma levels (with two additional miRNAs, miR-22-3p, miR-92a-3p) are recommended as potential diagnoses for schizophrenia, while bioinformatic analysis of target genes correlates with synaptic structure, function, and plasticity with varying neuroplasticity in schizophrenia. It is shown in accordance with its central role. Altogether, it seems to be among the most promising targets for the future development of molecular schizophrenia therapeutics because of its capacity to regulate an entire gene network involved in this single miRNA gene etiology. ${ }^{[5]}$

The cellular effects of risk-related miRNAs, hsa-miR-208b$3 p$, hsa miR-494-5p and hsa-miR-208a-3p, are aligned with primary etiological hypotheses of schizophrenia and should be investigated for possible pharmacological interventions as well as target genes of the three molecules. ${ }^{[18]}$ In several studies conducted in the context of schizophrenia, Sun et al. used qPCR to test nine miRNA expressions before and 6 weeks after antipsychotic treatment, found four levels of miRNA (miR-132, miR-181b, miR-30e and miR-432) in the plasma of schizophrenia patients. All four miRNAs have been shown to significantly decrease in patients after 6 weeks of treatment with any medication (olanzapine, quetiapine, ziprasidone or risperidone). Expression of miR-132, miR-181-b, miR-212 and miR-30e also significantly correlated with clinical score changes after treatment, and it was emphasized that miR181-b was the best independent predictor of response to treatment. In another study, miR-30a-5p and $-30 e-5 p$ in 30 schizophrenia patients were found to be significantly reduced in peripheral blood mononuclear cells compared to healthy individuals. ${ }^{[9]}$

\section{miRNA and Bipolar Disorder}

Bipolar disorder is a chronic, recurrent mood disorder with an incidence of $2.4 \%$ and a mood disorder that neutralizes the individual. Bipolar disorder is usually characterized by euthymic and intermittent mania/hypomania and depression attacks that are difficult to treat. ${ }^{[19]}$ It has been suggested that bipolar disorder results from abnormalities in synaptic and neuronal plasticity, and several candidate genes are involved in the pathophysiology of bipolar disorder. It has been suggested that miR-134 is involved in synaptic plasticity because it suppresses the translation of LIM Domain Kinase 1 (Limk1) -mRNA, which encodes a protein kinase that is heavily involved in dendritic spine development. miR-134 plasma levels can serve as a diagnostic biomarker for bipolar disorder and a prognostic biomarker for antipsychotic and mood stabilizer therapy. ${ }^{[4]}$ Compared to other common neuropsychiatric disorders, relatively little research has addressed the role of varying miRNA expression levels or premiRNA mutations in bipolar disorder. It is also worth noting that among the few published genome-wide association studies affecting miRNAs in bipolar disorder etiology, miR-137 is strongly associated with bipolar disorder, which is strongly debated with schizophrenia. In addition, a small study (29 bipolar disorder and 29 controls) reported high miR-34a expression in postmortem cerebellar tissues from bipolar disorder patients. ${ }^{[5]}$

It has been discovered that miR-7-5p can make upregulation differentiate patients with bipolar disorder from controls. Critically, miR-7 can prevent the repair of peripheral nerve damage by modulating the migration and proliferation of neural stem cells; also, miR-7 was detected by an increase in the neocortex of the superior temporal lobes affected by Alzheimer's disease. In a new study involving the rat model of herbicide-associated Parkinson disease, different miR-7 expressions were found in the brain and peripheral blood. The study revealed that upregulation of miR-7 in the brain, but downregulation of miR-7 in peripheral blood, as well as miR-7, and its concentration affects the pathophysiology of bipolar disorders by an autoregulatory mechanism. ${ }^{[20]}$ For miRNA studies in bipolar disorder, Azevedo et al. they selected 29 miRNA and among them, four miRNAs (miR-34a, miR-132, miR-133a and miR-212) were shown to be significantly low regulated in the anterior cingulate cortex $[A C C]$. Another study found increased expression of miR-149 in exosomes from the ACC of bipolar disorder subjects. The relative contribution of glian to neurons was found in the induction of miR-149 expression in the brain of bipolar disorder. ${ }^{[9]}$

Banigan et al. ${ }^{[9]}$ Reported upregulation of miR-29c. To examine the role of miRNAs in synaptic plasticity, synaptosomal miRNAs were studied in Brodmann's area [BA] BA10 of bipolar disorder subjects. Four are upregulated (miR-17-5p, miR-579, miR106b-5p and miR-29c-3p) and five are downregulated (miR145-5p, miR-485-5p, miR-370, miR- 500a-5p and miR-34a-5p) are shown. Upregulated miR-579 and downregulated miR34a expression changes were confirmed in bipolar disorder subjects.

\section{miRNA and Obsessive Compulsive Disorder}

Obsessive compulsive disorder (OCD) is a severe psychiatric illness with an incidence of 1-3\%, which lowers the individual's standard of living and functionality. The disorder is characterized by the presence of unwanted, permanent obsessions, which are often repeated in response to obsessions that cause anxiety or distress, along with compulsions that are ritual behaviors or mental actions, causing significant anxiety or distress. Obsessions can range from the fear of contamination to the experience of intrusive 
violent or obscene thoughts, and enforcement may include repeated control, washing, cleaning and counting. ${ }^{[21]}$ Within the framework of the OCD genome, studies have analyzed the genetic link between 9p24 and OCD and also identified several candidate genes. Few studies have emphasized the hereditary nature of such a disease. In particular, the onset of OCD and the symptoms of sequencing/symmetry of OCD are often hereditary. A high risk of developing OCD or some symptoms has also been reported in first degree relatives. OCD encoding the neuronal/epithelial high affinity glutamate transporter in the 9p24 chromosome region is known to be a candidate gene associated with SLC1A1/EAAC1. SLC1A1 is expressed in some functional areas of the brain and is involved in OCD in cortico-strial-thalamic-cortical circuits (CSTC). Many evidence shows a varying glutamate neurotransmission in CTSC circuits in OCD pathophysiology. The neuronal glutamate carrier gene (SLC1A1) is mainly expressed in CSTC circuits. To date, researchers have identified several areas of the genome that may contain disease genes, as well as many candidate genes. Such genes include SLC6A4, HTR2A, HTR2C, NTRK3 and SLITRK1. ${ }^{[22]}$ Muinos-Gimeno et al. ${ }^{[22]}$ found a role in the pathophysiology of anxiety disorders of the neurotrophin-3 receptor gene (NTRK3). Such a gene has a high variation in the miRNA recognition element of miR-485-3p, one of the most important miRNA associated with OCD. 23 OCD patients with an average age of $10.60 \pm 2.06$ years (range, $7-16$ years) and 40 controls with an average age of $11.25 \pm 2.93$ years ( $7-17$ years) to study miRNA levels in OCD for research purposes patient was included and increased levels of miR22-3p, miR24-3p, miR106b-5p, miR125b-5p and miR155a-5p were found in OCD patients compared to controls. In this study, increased miR125b levels were observed in OCD cases. Eipper-Mains et al. reported a relationship between miR125 levels and addiction disorders in mice. ${ }^{[23]}$

\section{miRNA and Alzheimer's Disease}

Alzheimer's disease (AD) is a progressive mental illness characterized by memory loss, multiple cognitive disorders and personality and behavioral changes. ${ }^{[2]} A D$ is an agerelated neurodegenerative disease, the most common senile dementia in the World. It is clinically characterized by progressive and irreversible cognitive dysfunction. The main pathological features of Alzheimer's disease are neurofibrillar tangles formed by phosphorylated Tau protein aggregates, and senile plaques formed by accumulation of the $\beta$ amyloid (A $\beta$ ) peptide. ${ }^{[25]}$ The imbalance between Tau phosphorylation and phosphorylation has been proposed as an alternative mechanism underlying the formation of senile plaques and AD development. miR-125b, miR-132, miR-26 and miR-146a trigger hyperphosphorylation of the Tau protein, which indirectly acts on different cellular factors. ${ }^{[26]}$ Higaki et al. ${ }^{[8]}$ to correlate differential expression of the miRNA-200 family (miRNA-200a, -141, -429, -200b, -200c) in Tg2576 (Tg2576 mice overexpress Amyloid Precursor Proteins (APP) protein (Swedish KM670/671NL mutation) in the first phases of
Alzheimer's disease) has done a study. Analysis of the total RNA microarray extracted from the cortical tissues of the mice showed that miRNA-200a, -141, -429, -200b and -200c were upregulated in Tg2576 mice only 10 months old.

Several miRNAs have been disclosed to play a role in different stages of $A D$ development, from regulation of $A B$ or Tau protein production to clearance. miR-106a/520c, miR-20, miR101, miR-16 and miR-153 can bind 3 'UTRs of APP resulting in lower expressions, both in vitro and in vivo. Alternatively, miR-29-a/b-1 and miR-29c have been found to regulate Beta Secretase Enzyme 1 (BACE1), the protease responsible for APP digestion. ${ }^{[26]}$

It has been suggested that miR-125b-5p can induce tau phosphorylation by inducing changes in tau kinase expression and activity, affecting p35, cdk5 and p44-42-MAPK signaling. miR-125b-5p is highly enriched in the brain and down-regulated in the serum, BOS and plasma of AD patients. Tan et al. ${ }^{[4]}$ (2014) identified miR-125b-5p as a biomarker with high sensitivity/specificity (80.8\%/68.3\%) for $A D$ and was negatively correlated with mini-mental state examination (MMSE) scores, a test for measuring cognitive functionality. The predicted target genes of miR-125b-5p include CDKN2A, SYN-2, and 15-LOX associated with glial proliferation, defective synaptogenesis/synaptic deficits, and neurotrophic deficits, respectively. 39 miRNA ADs containing the latest profiling data, such as miR-9,33 miR-15a, 31 miR-29,30 miR-101,31 miR106,37 miR107,36 miR-146,38 and miR-181c shows that it has specifically changed in the brain. This has been demonstrated independently in 2 or more studies. Interestingly, many of these candidates can play a direct role in modulating the expression of AD-related genes. ${ }^{[27]}$

miR-181c has been shown to be downregulated in the brain, CSF and blood of AD patients. In addition, the loss of this miRNA increases levels of Serine palmitoyltransferase (SPT), the first rate-limiting enzyme in de novo ceramide synthesis, which increases levels of amyloid precursor protein $(A \beta)$. Geekiyanage et al showed that serine palmitoyltransferase long chain 1 (SPTLC1) encoding a component of the SPT heterodimer is the direct miR-181c target. Downregulation of SPTLC1 with miR-181c overexpression has shown that it reduces Ap levels in primary astrocytes derived from transgenic mice expressing the human APP Swedish mutation. These data show an important role of miR-181c in AD and suggest this miRNA as a potential therapeutic target for this condition. ${ }^{[28]} \mathrm{miR}-146 \mathrm{a}-5 \mathrm{p}$ has an increased level of expression in both peripheral circulation and brains of AD patients. Subsequently, miR-146a-5p was associated with neuro-inflammation that promotes the degeneration of neurons.

Recently, increasing evidence has shown that dysfunction of miRNAs within neurons and varying expression of miRNAs are highly associated with the pathogenesis of neurodegenerative diseases. Therefore, regulation of miRNAs with exogenous interventions will provide a new perspective to explore the pathogenesis and neuropathology of Alzheimer's disease. 


\section{miRNA and Parkinson's Disease}

Parkinson's disease (PD) is the second most common neurodegenerative disease in the world after AD. PD affects $0.3 \%$ of the entire population and rises to $1 \%$ of the population over the age of 65 . Clinical symptoms of PD patients include rest tremor, muscle stiffness, bradykinesia and postural instability. Non-motor symptoms may occur in PD, including hyposmia, constipation, urinary dysfunction, depression, anxiety, and rapid eye movement sleep behavior disorder, before the appearance of motor symptoms. ${ }^{[29]} A$ possible role of the mir-181 family has also been proposed in Parkinson's disease. mir-181 $\mathrm{a}$ and $\mathrm{b}$ are expressed in Substantia Nigra and striatum and have been found to modulate the expression of genes associated with mitochondrial dependent cell death and autophagy (ie, B cell lymphoma 2 (BCL2), Myeloid Cell Leukemia 1, (MCL1)). In addition, PARK2 (Parkin), which is responsible for a monogenic form of Parkinson's disease and plays a role in the regulation of mitochondrial quality control and mitophage, is a direct target of miR-181a. In particular, the second pathways have been associated with Parkinson's pathogenesis and progression. ${ }^{[28]}$

Alpha Synuclein (a-Syn) is abundantly expressed in the brain of healthy individuals, and this interest has grown increasingly as a component of Lewy bodies in Parkinson's disease and other synucleinopathies. Indeed, genome-wide association studies (GWAS) are pathways affected by a-Syn expression, glycosphingolipid biosynthesis and ubiquitination of the protein, single nucleotide polymorphisms (SNP), and a series of microRNAs that are differently expressed in Parkinson's disease. The miR-7 well represents how a change in miRNA content directly affects a targeted mRNA. Patients with Parkinson's disease show reduced miR-7 levels in brain regions associated with disease neuropathology, especially substantia nigra, and depletion of this miRNA is functionally associated with a-Syn accumulation and greater neuron loss. In addition to miR-7, miR-153 also recognizes sequences in the 3 'UTR region of a-Syn so it works additionally to lower the gene. miR-7 causes a stronger down regulation on a-Syn compared to miR-153. ${ }^{[30]}$

Important research has been done to investigate early, specific and sensitive biomarkers for PD. However, despite the efforts, clinical biomarkers have not yet been established. The most commonly low regulated miRNAs found in PD are; miR-1263p, miR-133b, miR-1-3p, miR-214-3p, miR-221-3p, miR-29a-3p, miR-29b3p, miR-29c-3p, miR-30c-5p, miR-374a-5p and miR-19$3 p$. Parkinson's patients have irregular miR-30c-5p levels in the brain, a miRNA abundant in neuronal and glia cells. In addition, Martins et al. ${ }^{[4]}(2011)$ showed that miR-30c-5p interacts with proteins in the a-synuclein and glycosphingolipid biosynthesis and protein ubiquitination pathways, the latter of which are important for the ubiquitination of a-synuclein. Jiang $Y$ et al. ${ }^{[29]}$ (2019) reported that exosomal miR-137 is upregulated and plays a vital role in the induction of oxidative stress of neurons in PD. It has been found that miR-137 directly targets oxidation resistance 1 (OXR1) and negatively regulates its expression, thereby inducing oxidative stress in PD.

\section{RESULT}

miRNAs are aware of the events occurring in the cell as normal mechanical, physical and biochemical functions due to many psychiatric and medical diseases and are very promising as a biomarker for emerging disorders and neuropathological changes. Recent research findings show that the expression levels of some miRNAs deserve more research as temporary diagnostic biomarkers for neuropsychiatric disorders and neurodegenerative disorders, including major depressive disorder, bipolar disorder, schizophrenia and bipolar disorder. Studies on the irregular regulation of miRNAs and their use as a biomarker are increasing day by day. Also, miRNAs appear to be promising drug targets for the development of future psychiatric therapeutics. Understanding the role of irregular neuronal signaling, irregular miRNAs in neuronglia communication and neuroplasticity in such disorders will allow earlier, more accurate and more precise diagnosis for affected individuals. However, targeting miRNAs appears to represent a valuable and innovative approach that can overcome currently existing drug limitations and provide a new and powerful perspective on the neurobiology and transmission of these diseases over generations.

\section{ETHICAL DECLARATIONS}

Status of Peer-review: Externally peer-reviewed. Conflict of Interest Statement: The authors have no conflicts of interest to declare.

Financial Disclosure: The authors declared that this study has received no financial support.

Author Contributions: All of the authors declare that they have all participated in the design, execution, and analysis of the paper, and that they have approved the final version.

\section{REFERENCES}

1. Parikh NI, Vasan RS. Assessing the clinical utility of biomarkers in medicine. Biomark Med 2007; 1: 419-36.

2. Mosallaei M, Ehtesham N, Rahimirad S, Saghi M, Vatandoost N, Khosravi S. PBMCs: a new source of diagnostic and prognostic biomarkers. Arch Physiol Biochem 2020; 1-7. doi:10.1080/13813455.2020.1752257

3. Zhang HP, Liu XL, Chen JJ, et al. Circulating microRNA 134 sheds light on the diagnosis of major depressive disorder. Transl Psychiatry 2020; 10: 95. doi:10.1038/s41398-020-0773-2.

4. van den Berg MMJ, Krauskopf J, Ramaekers JG, Kleinjans JCS, Prickaerts J Briedé JJ. Circulating microRNAs as potential biomarkers for psychiatric and neurodegenerative disorders. Prog Neurobiol 2020; 185: 101732. doi:10.1016/j.pneurobio.2019.101732

5. Gurwitz D. Genomics and the future of psychopharmacology: MicroRNAs offer novel therapeutics. Dialogues Clin Neurosci 2019; 21: 131-48. doi:10.31887/DCNS.2019.21.2/dgurwitz

6. Kiltschewskij DJ, Geaghan MP, Cairns MJ.Characterising theTranscriptional and Translational Impact of the Schizophrenia-Associated miR-1271-5p in Neuronal Cells. Cells 2020; 9: 1014.

7. Fregeac J, Moriceau S, Poli A, Nguyen LS, Oury F, Colleaux L. Loss of the neurodevelopmental disease-associated gene miR-146a impairs neural progenitor differentiation and causes learning and memory deficits. Molecular autism 2020; 11: 1-14. 
8. Silvestro S, Bramanti $\mathrm{P}$, Mazzon E. Role of miRNAs in Alzheimer's Disease and Possible Fields of Application. Int J Mol Sci 2019; 20: 3979. doi:10.3390/ijms20163979

9. Roy B, Yoshino Y, Allen L, Prall K, Schell G, Dwivedi Y. Exploiting circulating MicroRNAs as biomarkers in psychiatric disorders. Mol Diagn Ther 2020; 24: 279-8. doi:10.1007/s40291-020-00464-9

10. Ferrúa CP, Giorgi R, da Rosa LC, et al. MicroRNAs expressed in depression and their associated pathways: A systematic review and a bioinformatics analysis. J Chem Neuroanat 2019; 100: 101650. doi:10.1016/j. jchemneu.2019.101650.

11. Wang L, Zhang L. Circulating exosomal miRNA as diagnostic biomarkers of neurodegenerative diseases. Front Mol Neurosci 2020; 13: 53. doi:10.3389/fnmol.2020.00053

12. Malan-Müller S, Hemmings $S$. The big role of small RNAs in anxiety and stress-related disorders. Vitamins and hormones. Elsevier 2017; 103: 85129.

13. Ciebiera M, Włodarczyk M, Zgliczyński S, Łoziński T, Walczak K, Czekierdowski A. The role of miRNA and related pathways in pathophysiology of uterine fibroids-from bench to bedside. Int J Mol Sci 2020; 21: 3016. doi:10.3390/ijms21083016.

14. Fries GR, Zhang W, Benevenuto D, Quevedo J. MicroRNAs in major depressive disorder. Adv Exp Med Biol 2019; 1118: 175-90. doi:10.1007/978-3-030-05542-4_9.

15. Allen L, Dwivedi Y. MicroRNA mediators of early life stress vulnerability to depression and suicidal behavior. Mol Psychiatry 2020; 25: 308-20. Doi.10.1038/s41380-019-0597-8.

16. Goto $Y$, Yang CR, Otani S. Functional and dysfunctional synaptic plasticity in prefrontal cortex: roles in psychiatric disorders. Biol Psychiatry 2010; 67: 199-207. doi:10.1016/j.biopsych.2009.08.026.

17. Smigielski L, Jagannath V, Rössler W, Walitza S, Grünblatt E. Epigenetic mechanisms in schizophrenia and other psychotic disorders: a systematic review of empirical human findings. Mol Psychiatry 2020; 25: 1718-48. doi:10.1038/s41380-019-0601-3.

18. Cao H, Baranova A, Yue W, et al. MiRNA-coordinated schizophrenia risk network cross-talk with cardiovascular repair and opposed gliomagenesis. Front Genet 2020; 11: 149. doi:10.3389/fgene.2020.00149.

19. Kidnapillai S, Wade B, Bortolasci CC, et al. Drugs used to treat bipolar disorder act via microRNAs to regulate expression of genes involved in neurite outgrowth. J Psychopharmacol 2020; 34: 370-9. doi:10.1177/0269881119895534.

20. Lee $S$, Lu R, Wang $L$, et al. Serum miRNA as a possible biomarker in the diagnosis of bipolar II disorder. Sci Rep 2020: 10; 1131. doi:10.1038/ s41598-020-58195-0.

21. Jansen M, Overgaauw S, De Bruijn ERA. Social cognition and obsessivecompulsive disorder: a review of subdomains of social functioning. Front Psychiatry 2020; 11: 118. doi:10.3389/fpsyt.2020.00118.

22. Privitera AP, Distefano R, Wefer HA, Ferro A, Pulvirenti A, Giugno R. OCDB: a database collecting genes, miRNAs and drugs for obsessive-compulsive disorder. Database (Oxford). 2015; 2015: bav069. doi:10.1093/database/ bav069.

23. Kandemir H, Erdal ME, Selek S, et al. Microribonucleic acid dysregulations in children and adolescents with obsessive-compulsive disorder Neuropsychiatr Dis Treat 2015; 11: 1695-701.

24. Reddy AP, Ravichandran J, Carkaci-Salli N. Neural regeneration therapies for Alzheimer's and Parkinson's disease-related disorders. Biochim Biophys Acta Mol Basis Dis 2020; 1866: 165506. doi:10.1016/j.bbadis.2019.06.020.

25. Kuang $\mathrm{H}$, Tan $\mathrm{CY}$, Tian $\mathrm{HZ}$, et al. Exploring the bi-directional relationship between autophagy and Alzheimer's disease. CNS Neurosci Ther 2020; 26: 155-66. doi:10.1111/cns.13216.

26. Conti I, Varano G, Simioni C, et al. miRNAs as Influencers of cell-cell communication in tumor microenvironment. Cells 2020; 9: 220. doi: $10.1111 / \mathrm{cns} .13216$

27. Souza VC, Morais GS Jr, Henriques AD, et al. Whole-blood levels of microRNA-9 are decreased in patients with late-onset alzheimer disease. Am J Alzheimers Dis Other Demen 2020; 35: 1533317520911573. doi:10.1177/1533317520911573.
28. Indrieri A, Carrella S, Carotenuto P, Banfi S, Franco B. The pervasive role of the miR-181 family in development, neurodegeneration, and cancer. Int J Mol Sci 2020; 21: 2092. doi:10.3390/ijms21062092.

29. Yu H, Sun T, An J, et al. Potential roles of exosomes in parkinson's disease: from pathogenesis, diagnosis, and treatment to prognosis. Front Cell Dev Biol 2020; 8: 86. doi:10.3389/fcell.2020.00086.

30. Titze-de-Almeida SS, Soto-Sánchez C, Fernandez E, Koprich JB, Brotchie $J M$, Titze-de-Almeida R. The promise and challenges of developing miRNA-based therapeutics for Parkinson's Disease. Cells 2020; 9: 841. doi:10.3390/cells9040841. 